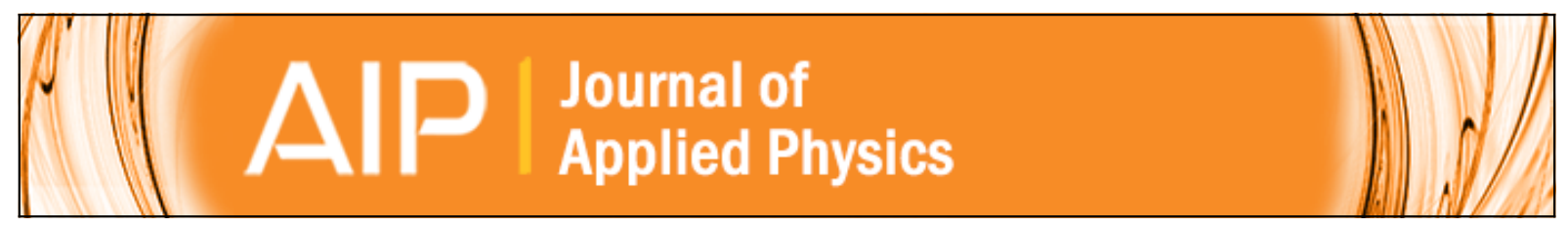

\title{
Nonlinear piezoelectricity in electroelastic energy harvesters: Modeling and experimental identification
}

Samuel C. Stanton, Alper Erturk, Brian P. Mann, and Daniel J. Inman

Citation: Journal of Applied Physics 108, 074903 (2010); doi: 10.1063/1.3486519

View online: http://dx.doi.org/10.1063/1.3486519

View Table of Contents: http://scitation.aip.org/content/aip/journal/jap/108/7?ver=pdfcov

Published by the AIP Publishing

\section{Articles you may be interested in}

Experimental verification of a bridge-shaped, nonlinear vibration energy harvester Appl. Phys. Lett. 105, 203901 (2014); 10.1063/1.4902116

Micro-fabricated lead zirconate titanate bent cantilever energy harvester with multi-dimensional operation Appl. Phys. Lett. 102, 043901 (2013); 10.1063/1.4789754

Energy harvesting properties of all-thin-film multiferroic cantilevers

Appl. Phys. Lett. 99, 203506 (2011); 10.1063/1.3662037

Sensor shape design for piezoelectric cantilever beams to harvest vibration energy

J. Appl. Phys. 108, 014901 (2010); 10.1063/1.3457330

Piezoelectric energy harvesting using shear mode 0.71 Pb ( Mg $1 / 3 \mathrm{Nb} 2 / 3$ ) O $3-0.29 \mathrm{PbTiO} 3$ single crystal cantilever

Appl. Phys. Lett. 96, 083502 (2010); 10.1063/1.3327330

\section{MIT LINCOLN} LABORATORY CAREERS

Discover the satisfaction of innovation and service to the nation
- Space Control

- Air \& Missile Defense

- Communications Systems \& Cyber Security

- Intelligence, Surveillance and

Reconnaissance Systems

$$
\begin{aligned}
& \text { - Advanced } \\
& \text { Electronics } \\
& \text { - Tactical Systems } \\
& \text { " Homeland } \\
& \text { Protection } \\
& \text { - Air Traffic Control }
\end{aligned}
$$

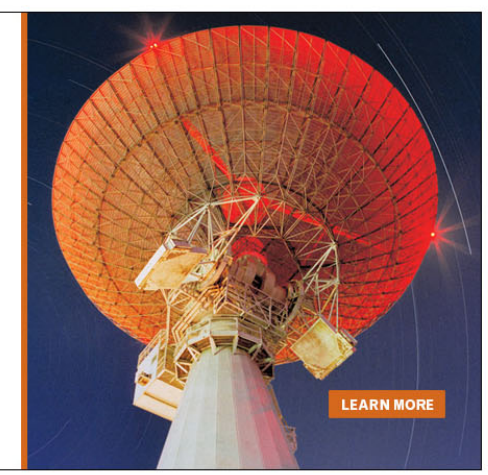




\title{
Nonlinear piezoelectricity in electroelastic energy harvesters: Modeling and experimental identification
}

\author{
Samuel C. Stanton, ${ }^{1, a)}$ Alper Erturk, ${ }^{2}$ Brian P. Mann, ${ }^{1}$ and Daniel J. Inman ${ }^{2}$ \\ ${ }^{1}$ Department of Mechanical Engineering, Nonlinear Dynamical Systems Laboratory, Duke University, \\ Durham, North Carolina 27708, USA \\ ${ }^{2}$ Department of Mechanical Engineering, Center for Intelligent Material Systems and Structures, \\ Virginia Tech, Blacksburg, Virginia 24061, USA
}

(Received 8 June 2010; accepted 7 August 2010; published online 5 October 2010)

\begin{abstract}
We propose and experimentally validate a first-principles based model for the nonlinear piezoelectric response of an electroelastic energy harvester. The analysis herein highlights the importance of modeling inherent piezoelectric nonlinearities that are not limited to higher order elastic effects but also include nonlinear coupling to a power harvesting circuit. Furthermore, a nonlinear damping mechanism is shown to accurately restrict the amplitude and bandwidth of the frequency response. The linear piezoelectric modeling framework widely accepted for theoretical investigations is demonstrated to be a weak presumption for near-resonant excitation amplitudes as low as $0.5 \mathrm{~g}$ in a prefabricated bimorph whose oscillation amplitudes remain geometrically linear for the full range of experimental tests performed (never exceeding $0.25 \%$ of the cantilever overhang length). Nonlinear coefficients are identified via a nonlinear least-squares optimization algorithm that utilizes an approximate analytic solution obtained by the method of harmonic balance. For lead zirconate titanate (PZT-5H), we obtained a fourth order elastic tensor component of $c_{1111}^{p}=-3.6673 \times 10^{17} \mathrm{~N} / \mathrm{m}^{2}$ and a fourth order electroelastic tensor value of $e_{3111}=1.7212$ $\times 10^{8} \mathrm{~m} / \mathrm{V}$. () 2010 American Institute of Physics. [doi:10.1063/1.3486519]
\end{abstract}

\section{INTRODUCTION}

Flexible piezoelectric devices have a long history of successful research pertaining to actuation and sensing technology for smart material systems. Most recently, their utility has been promulgated throughout a wide body of literature focusing on converting the sensor problem to one of vibratory energy harvesting instead. Vibratory energy harvesting is the process by which self-reliant electronic systems are realized by transforming environmental excitation sources into usable electricity. ${ }^{1,2}$ Success in this endeavor is largely attributed to reduced power demands for electronic devices to the micro- and milliwatt scale. ${ }^{3,4}$ In addition, selfsustaining energy harvesting systems are viewed as an enabling technology for cost-effective wireless sensor networks whose proposed application venues (i.e., remote-area, in vivo, bridges, etc.) render regular maintenance and battery replacement problematic. ${ }^{5-8}$ To date, most piezoelectric harvesters presume linear response and thus seek electromechanical resonance to extract maximum energy. ${ }^{2,9-11}$ However, considering many environmental excitation sources are instead broadband, strategies ranging from control theoretic $^{12-14}$ to purposeful inclusion of nonlinearity ${ }^{15-22}$ are gaining momentum. For very low excitation levels for all such harvesters, linear behavior of the piezoelectric components is an accurate presumption.

Linear modeling of piezoelectric harvesters is accomplished according to the variational framework established by Hagood et al. ${ }^{23}$ for electroelastic actuators and sensors. Wang and $\mathrm{Cross}^{24}$ later discussed how polarization of bi-

${ }^{\text {a)} E l e c t r o n i c ~ m a i l: ~ s a m u e l . s t a n t o n @ d u k e . e d u . ~}$ morph piezoelectric cantilevers connected series and parallel effects the linear piezoelectric constitutive equations. Sodano et $a l^{10}{ }^{10}$ were among the first to apply these results to the analysis of an energy harvester and du Toit et al. ${ }^{9}$ updated the model to include effects of end masses with rotary inertia. In a series of papers, Erturk and Inman ${ }^{25,26}$ studied in detail linear models for both unimorph and bimorph cantilevered harvesters with both a translational and a small rotary base excitation with further analysis of resonance shifts brought about by electrical impedance interactions. Furthermore, the same authors addressed errors in simplified single degree of freedom models ${ }^{27}$ and other unsound modeling practices that began to emerge in the literature. ${ }^{28,29}$

Nonlinear behavior within piezoceramics and associated influence on the energy harvesting problem, however, has only begun to receive attention. Hu et al. ${ }^{30}$ numerically studied the large amplitude hardening response of a piezoelectric plate under thickness/shear vibration. The physical basis for their model was the cubic theory of nonlinear electroelasticity ${ }^{31-33}$ with up to fourth order elastic effects but higher order coupling and electrical effects neglected due to the weak nature of the electric fields. The same electroelasticity model was previously applied by Wolf and Gottlieb $^{34,35}$ to describe the large amplitude motion of atomic force microscopes with later experimental validation by Usher and Sim. ${ }^{36}$ Triplett and Quinn ${ }^{37}$ theoretically studied nonlinear piezoelectric energy harvester models with nonlinear coupling effects as observed by Crawley and Anderson ${ }^{38}$ and Crawley and Lazarus. ${ }^{39}$ However, the nonlinearity in the coupling in Refs. 38 and 39 was due to induced actuation strain as a consequence of large electric fields as explained and modeled by Tiersten. ${ }^{33}$ Whereas the insignificance of the 


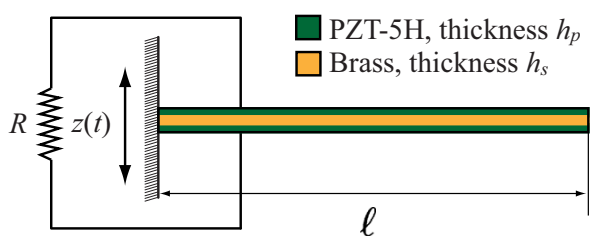

FIG. 1. (Color online) Illustration of a prototypical piezoelectric energy harvester connected in series to a resistive load.

electric field prompted $\mathrm{Hu}$ et al. to neglect coupling nonlinearities, von Wagner and Hagedorn ${ }^{40,41}$ and Samal et al. ${ }^{42}$ have demonstrated significant nonlinear effects in piezoelectric actuators experiencing weak electric fields. Considering this observation, Stanton and $\mathrm{Mann}^{43}$ recently derived and experimentally verified governing equations for the large amplitude motion of prototypical energy harvesting beams carrying an end mass. The model included piezoelectric nonlinearities by augmenting the model in Ref. 30 to reflect higher order coupling effects as observed in Ref. 40. However, there is some uncertainty in the estimates for the nonlinear tensor components due to a multitude of sources contributing to nonlinear behavior in the experimental device: large amplitude mechanical motion, end mass inertia, viscoelasticity in the substrate, heterogeneous piezoceramic distribution, possible unmodeled nonlinear damping, and nonlinear piezoelectricity.

To address this problem, the objective of this paper is to focus on manifestation of nonlinearity in a cantilevered piezoelectric harvester with geometrically linear behavior (small amplitude oscillations) and no end mass. This essentially isolates piezoelectric effects as the principal source of nonlinearity. The results of our investigation are organized as follows: In Sec. II, we use energy methods to derive a model based on the analysis in Ref. 43, but with the simplifying assumption of linear strain. As a result, the nonlinear terms only reflect piezoelectric material nonlinearities. Sec. III describes the experimental set up and frequency content of the data, which motivates inclusion of a nonlinear damping term. Following parameter identification, Sec. IV provides a summary and conclusions.

\section{THEORETICAL MODELING}

Consider a symmetric bimorph cantilever connected in series to a resistive load as shown in Fig. 1. The cantilever extends a length $\ell$ from the clamp location and has piezoceramics with density $\rho_{p}$ of individual thickness $h_{p}$ symmetrically laminated above and below an electrically inactive substrate of density $\rho_{s}$ with thickness $h_{s} / 2$ on either side of the neutral bending axis. Also, the piezoceramic is homogeneous along the length of the cantilever. Nonlinear electroelasticity is postulated to develop in accordance with the following nonlinear constitutive equations: $:^{31,32,40}$

$$
\begin{aligned}
T_{1}= & c_{11}^{p} S_{1}+c_{111}^{p} S_{1}^{2}+c_{1111}^{p} S_{1}^{3}-e_{31} E_{3}-e_{311} S_{1} E_{3} \\
& -e_{3111} S_{1}^{2} E_{3},
\end{aligned}
$$

$$
D_{3}=e_{31} E_{3}+e_{311} S_{1} E_{3}+e_{3111} S_{1}^{2} E_{3}+\epsilon_{33}^{s} E_{3},
$$

where $T_{1}$ and $S_{1}$ represent the stress and strain along the length of the beam while $D_{3}$ and $E_{3}$ indicate the electric displacement and electric field that develops through the thickness of the piezoelectric laminates. The material constants $c_{11}^{p}, c_{111}^{p}$, and $c_{1111}^{p}$ and $e_{31}, e_{311}$, and $e_{3111}$ are second, third, and fourth order elastic and electroelastic tensor components, respectively. The elastic tensor values are as measured in a zero electric field. The tensor subscripts $(1,2,3)$ correspond to the Cartesian system $(x, y, z)$. The electric permittivity at zero strain is given by $\epsilon_{33}^{s}$. In Eqs. (1a) and (1b), the weak nature of the electric fields motivates exclusion of nonlinear dielectric effects and electrostriction. Also, time dependent dissipative effects, viscoelasticity, and gravity induced asymmetry are presumed insignificant. While the analysis due to Guyomar et al. ${ }^{44}$ considered a second order expansion of the linear constitutive equations, the third order expansion and nonlinear coupling in our model is selected so that nonlinear terms do not cancel in the derivation of the equations of motion due to the symmetry of the cantilever. Considering that the experimental model readily exhibits a nonlinear frequency response and presuming perfect symmetry, the model would be incapable of predicting nonlinear phenomena otherwise.

Mechanical strain is modeled by Euler-Bernoulli beam theory such that $S_{1}=-z w^{\prime \prime}$, where $w=w(x, t)$ is the deflection of the beam and the prime notation ( )' is shorthand for $\partial / \partial x$. Finally, the electrical field is presumed to be linear and uniform through the thickness of each laminate so that the solution to Gauss' equation is truncated to $E_{3}= \pm \dot{\lambda} / 2 h_{p}$, where $\lambda$ is the flux linkage coordinate and the sign of the field is opposite for the upper and lower laminates.

Flux linkage coordinates facilitate a unified variational derivation for the electromechanical dynamics of the entire energy harvesting device. ${ }^{45}$ We note that a nonuniform electric field assumption is worthy of future consideration in light of the analysis due to Tabesh and Fechette ${ }^{46}$ and Low and Guo. ${ }^{47}$

\section{A. Nonlinear electroelastic energy expressions}

The kinetic energy within the harvester is distributed along the length of the beam as

$$
T=\frac{1}{2} m \int_{0}^{\ell}(\dot{w}+\dot{z})^{2} d x
$$

where $m=\rho_{s} h_{s}+2 \rho_{p} h_{p}$ is the mass per unit length of the beam and $z$ is the velocity of the base. The total conservative bending potential through each layer of the bimorph is given by

$$
U=\frac{1}{2} E I_{s} \int_{0}^{\ell} w^{\prime \prime 2} d x+\frac{1}{2} \int_{V_{p}}\left(T_{1} S_{1}-D_{3} E_{3}\right) d V_{p},
$$

where $V_{p}$ is the total volume of the piezoceramic laminates and $E I_{S}$ is the substrate bending rigidity. Upon integrating over the appropriate dimensions, Eq. (3) becomes 


$$
\begin{aligned}
U= & \frac{1}{2} \int_{0}^{\ell}\left[E I w^{\prime \prime 2}+\frac{1}{6} G w^{\prime \prime 4}-2\left(\hat{\theta} w^{\prime \prime}+\frac{1}{6} \hat{\varphi} w^{\prime \prime 3}\right) \dot{\lambda}\right] d x \\
& -\frac{1}{2} C \dot{\lambda}^{2},
\end{aligned}
$$

where the coefficients are defined as

$$
\begin{aligned}
& E I=E I_{s}+\frac{1}{6} c_{11}^{p} b h_{p}\left(4 h_{p}^{2}+6 h_{p} h_{s}+3 h_{s}^{2}\right), \\
& G=\frac{3}{20} c_{1111}^{p} b h_{p}\left(16 h_{p}^{4}+40 h_{p}^{3} h_{s}+40 h_{p}^{2} h_{s}^{2}+20 h_{p} h_{s}^{3}+5 h_{s}^{4}\right),
\end{aligned}
$$

$$
\hat{\theta}=\frac{1}{2} e_{31} b\left(h_{p}+h_{s}\right)
$$

$$
\hat{\varphi}=\frac{3}{4} e_{3111} b\left(h_{p}+h_{s}\right)\left(2 h_{p}^{2}+2 h_{p} h_{s}+h_{s}^{2}\right),
$$

and

$$
C=\frac{\epsilon_{33}^{s} b \ell}{2 h_{p}} .
$$

The first term in Eq. (4) is the linear bending energy, the term proportional to $\hat{\theta}$ is the linear coupling coefficient, and $C$ is the series capacitance of both piezoelectric laminates. Nonlinear piezoelasticity is modeled by the second term and nonlinear coupling is proportional to $\hat{\varphi}$. Due to the symmetry of the bimorph configuration, third order nonlinearities from terms proportional to $c_{111}^{p}$ and $e_{311}$ vanish. As can be inferred from the potential field, sources of nonlinearity in a bimorph piezoelectric device will primarily be cubic.

\section{B. Derivation of the governing nonlinear partial differential equations}

To derive the governing equations for the harvester, Hamilton's principle requires the variation in the sum of the Lagrangian $\mathcal{L}=T-U$ and nonconservative work terms to reach a stationary value between two instances in time, i.e.,

$$
0=\int_{t_{0}}^{t_{1}} \delta \mathcal{L}+\delta W d t
$$

where $\delta$ is a variational derivative, ${ }^{45}$

$$
\delta \mathcal{L}\left(\dot{w}, \dot{\lambda}, w^{\prime \prime}\right)=\frac{\partial \mathcal{L}}{\partial \dot{w}} \delta \dot{w}+\frac{\partial \mathcal{L}}{\partial \dot{\lambda}} \delta \dot{\lambda}+\frac{\partial \mathcal{L}}{\partial w^{\prime \prime}} \delta w^{\prime \prime},
$$

and the variation in the nonconservative work is

$$
\delta W=-c \dot{w} \delta w-\frac{\dot{\lambda}}{R} \delta \lambda .
$$

In Eq. (8), above, dissipative structural effects are modeled as proportional damping in the first term and the second term models the power delivered to a resistive load $R$. Later, in Sec. III, the presumption of linear damping alone will be revisited. Applying methods of Calculus of Variations and collecting terms common in virtual motions of deflection and flux linkage coordinates yields the variational indicator

$$
\begin{aligned}
0= & \int_{t_{1}}^{t_{2}}\left\{\left[-\frac{\partial}{\partial t}\left(\frac{\partial \mathcal{L}}{\partial \dot{w}}\right)-c \dot{w}\right.\right. \\
& \left.+\frac{\partial^{2}}{\partial x^{2}}\left(\frac{\partial \mathcal{L}}{\partial w^{\prime \prime}}\right)\right] \delta \omega+\left[-\frac{\partial}{\partial t}\left(\frac{\partial \mathcal{L}}{\partial \dot{\lambda}}\right)-\frac{\dot{\lambda}}{R}\right] \delta \lambda \\
& \left.+\left.\left[-\frac{\partial}{\partial x}\left(\frac{\partial \mathcal{L}}{\partial w^{\prime \prime}}\right)\right] \delta w\right|_{0} ^{L}+\left.\frac{\partial \mathcal{L}}{\partial w^{\prime \prime}} \delta w^{\prime}\right|_{0} ^{L}\right\} d t .
\end{aligned}
$$

By performing the partial derivatives within the variational indicator and considering Eqs. (2), (4), and (8), the following nonlinear partial differential equations are obtained:

$$
\begin{aligned}
& m \ddot{w}+ c \dot{w}+E I w^{i v}+G w^{\prime \prime}\left(w^{\prime \prime} w^{i v}+2 w^{\prime \prime \prime 2}\right) \\
&-\left(\hat{\theta}\left[\hat{\delta}^{\prime}(x)-\hat{\delta}^{\prime}(x-L)\right]+\hat{\varphi}\left[w^{\prime \prime} w^{i v}+w^{\prime \prime \prime 2}\right]\right) \dot{\lambda}=m \ddot{z}, \\
& C \ddot{\lambda}+\frac{1}{R} \dot{\lambda}+\int_{0}^{L}\left(\hat{\theta}+\frac{1}{2} \hat{\varphi} w^{\prime \prime 2}\right) \dot{w}^{\prime \prime} d x=0,
\end{aligned}
$$

where time dependent linear coupling has been inserted into the equation for mechanical displacement with the Dirac delta function $\hat{\delta}(x)$. By removing nonlinear piezoelectric effects proportional to $G$ and $\hat{\varphi}$, the linear model of Erturk and Inman $^{25}$ is recovered.

\section{Distributed parameters model}

To facilitate parameter identification for near-resonant excitations, Galerkin's method is applied to transform the nonlinear partial differential equations in Eqs. (10) and (11) into a set of coupled nonlinear ordinary differential equations. In particular, we separate the transverse deflection $w(x, t)$ into a summation of $N$ generalized displacements $x_{n}(t)$ and orthogonal basis function $\phi_{n}(x)$ as

$$
w(x, t)=\sum_{n=1}^{\infty} x_{n}(t) \phi_{n}(x) .
$$

The orthogonal basis functions utilized in this analysis are the linear basis functions for Euler-Bernoulli beams with fixed-free boundary conditions ${ }^{48}$ with the additional condition of mass normalization according to

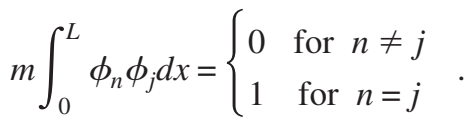

Inserting Eq. (12) into Eqs. (10) and (11), multiplying Eq. (10) by $\phi_{n}(x)$ and subsequently integrating over the length of the beam yields ordinary differential equations of motion (for $n=1,2,3, \ldots, \mathrm{N})$

$$
\begin{gathered}
\ddot{x}_{n}+2 \zeta \omega_{n} \dot{x}_{n}+\omega_{n}^{2} x_{n}+\sum_{j, k, \ell=1}^{N} \alpha_{n j k \ell} x_{j} x_{k} x_{\ell} \\
-\left(\theta_{n}+\sum_{j, k=1}^{N} \varphi_{n j k} x_{j} x_{k}\right) \dot{\lambda}=\Gamma_{n} \ddot{z},
\end{gathered}
$$




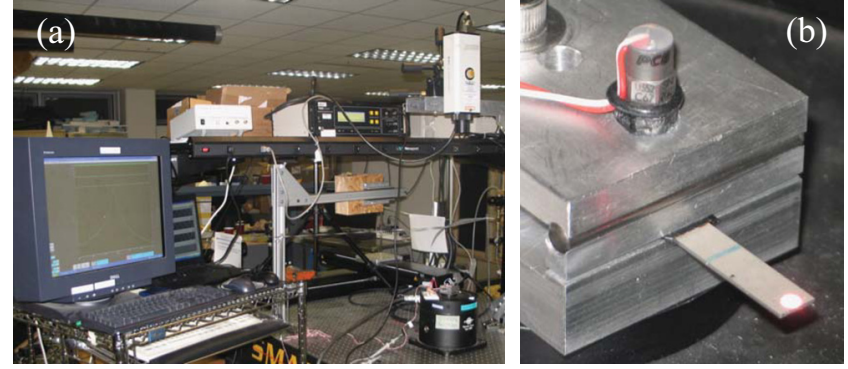

FIG. 2. (Color online) (a) Experimental setup used for the electromechanical measurements of the bimorph cantilever and (b) a close-up view of the cantilever, its clamp and the accelerometer. All experimental tests were performed at the Center for Intelligent Material Systems and Structures at Virgina Tech.

$$
C \ddot{\lambda}+R^{-1} \dot{\lambda}+\left(\theta_{n}+\sum_{j, k=1}^{N} \kappa_{n j k} x_{j} x_{k}\right) \dot{x}_{n}=0,
$$

where the coefficients are defined by

$$
\begin{aligned}
& \alpha_{n j k \ell}=G \int_{0}^{L} \phi_{n}\left[\phi_{j}^{\prime \prime}\left(\phi_{k}^{\prime \prime} \phi_{\ell}^{i v}+2 \phi_{k}^{\prime \prime \prime} \phi_{\ell}^{\prime \prime \prime}\right)\right] d x, \\
& \theta_{n}=\hat{\theta} \phi_{n}^{\prime}(L), \\
& \varphi_{n j k}=\hat{\varphi} \int_{0}^{L} \phi_{n}\left(\phi_{j}^{\prime \prime} \phi_{k}^{i v}+\phi_{j}^{\prime \prime \prime} \phi_{k}^{\prime \prime \prime}\right) d x, \\
& \Gamma_{n}=m \int_{0}^{L} \phi_{n} d x,
\end{aligned}
$$

and

$$
\kappa_{n j k}=\frac{1}{2} \hat{\varphi} \int_{0}^{L} \phi_{n}^{\prime \prime} \phi_{j}^{\prime \prime} \phi_{k}^{\prime \prime} d x .
$$

Again, due to the presumed symmetry of the problem, no quadratic nonlinearities result from the conservative potential field in Eq. (4). Sources of elastic nonlinearity are cubic and the nonlinear coupling is third order as well. In the linear case, coupling between the mechanical and electrical domains is proportional to the modal velocity. Equations (10) and (11), however, indicate that upon consider piezoelectric nonlinearities, the modal displacement also plays a role transferring energy to the impedance load. Stanton and Mann $^{43}$ showed that for large amplitude motion a coupling component proportional to the modal amplitude squared is present even when piezoelectric nonlinear effects are ignored. The same result has recently been obtained by Massana and Daqaq ${ }^{49}$ for the large amplitude motion of axially loaded piezoelectric harvesters with clamped-clamped boundary conditions and no piezoelectric material nonlinearity.

\section{EXPERIMENTAL INVESTIGATION}

The experimental setup used in this paper is shown in Fig. 2(a). The bimorph cantilever (T226-H4-203X, Piezo Systems Inc.) is clamped onto an electromagnetic shaker
TABLE I. Geometric and material properties of the PZT-5H bimorph cantilever.

\begin{tabular}{lcc}
\hline \hline Parameter & $\begin{array}{c}\text { Piezoceramic } \\
(\text { PZT-5H) }\end{array}$ & $\begin{array}{c}\text { Substructure } \\
\text { (brass) }\end{array}$ \\
\hline Length $\ell(\mathrm{mm})$ & 24.06 & 24.06 \\
Width $b(\mathrm{~mm})$ & 6.4 & 6.4 \\
Thickness $\left(h_{p}, h_{s}\right)(\mathrm{mm})$ & $0.265(\mathrm{each})$ & 0.140 \\
Mass density $\left(\rho_{p}, \rho_{s}\right)\left(\mathrm{kg} / \mathrm{m}^{3}\right)$ & 7500 & 9000 \\
Elastic modulus $\left(c_{11}^{p}, E\right)(\mathrm{GPa})$ & 60.6 & 105 \\
Piezoelectric constant $\left(e_{31}\right)\left(\mathrm{C} / \mathrm{m}^{2}\right)$ & -16.6 & $\cdots$ \\
Permittivity constant $\epsilon_{33}^{s}(\mathrm{nF} / \mathrm{m})$ & 25.55 & $\cdots$ \\
\hline \hline
\end{tabular}

(B\&K 4808) as displayed in Fig. 2(b). The bimorph consists of two oppositely poled lead zirconate titanate (PZT-5H) layers bracketing a brass layer that provides electrical conductivity between the piezoelectric elements (series connection). The properties of the cantilever are listed in Table I along with the plane-stress elastic, piezoelectric and dielectric properties of the piezoceramic.

A small accelerometer (PCB Piezotronics U352C67) is attached via wax close to the root of the cantilever on the clamp [Fig. 2(b)]. The transverse tip velocity response of the cantilever relative to the fixed reference frame is measured using a laser vibrometer (Polytec OFV353 laser head with OFV3001 vibrometer) by attaching a small reflector tape at the cantilever tip. Chirp excitation of low acceleration level (burst type with ten averages) is sent from the data acquisition system (SigLab 20-42) in order to extract the linear electromechanical frequency response functions (FRFs) for a set of resistors. After obtaining from the linear electromechanical FRFs (voltage-to-base acceleration and tip velocityto-base acceleration) that the resonance frequency for a $100 \mathrm{k} \Omega$ load is around $542 \mathrm{~Hz}$, the focus is placed on the following frequencies for the high-sampling-frequency time domain measurements: 530, 535, 540, 545, and $550 \mathrm{~Hz}$. Base excitation was harmonic of the form $\ddot{z}=Z_{e} \cos \Omega_{e} t$, where $Z_{e}$ is the base acceleration amplitude and $\Omega_{e}$ is the excitation frequency.

For 11 different values of base acceleration the steady state tip velocity and voltage output are recorded with a sampling frequency of $50 \mathrm{kHz}$. The base acceleration values were held constant for each excitation frequency since nonlinear responses are sensitive to variations in excitation amplitude. Base acceleration levels were $60 \mathrm{mg}, 145 \mathrm{mg}, 230$ $\mathrm{mg}, 310 \mathrm{mg}, 430 \mathrm{mg}, 560 \mathrm{mg}, 840 \mathrm{mg}, 1.12 \mathrm{~g}, 1.4 \mathrm{~g}, 1.7 \mathrm{~g}$, and $2 \mathrm{~g}$, where $\mathrm{g}=9.81 \mathrm{~m} / \mathrm{s}^{2}$. The time histories are recorded after the electromechanical system reached the steady state. For convenience, all measurements are taken for a fixed resistive load of $100 \mathrm{k} \Omega$.

Figure 3 illustrates immediate discrepancy observed between linear modeling and steady state experimental data. The linear FRF (Ref. 25) is in excellent agreement with experimental observations for the lowest excitation value of $Z_{e}=60 \mathrm{mg}$. However, for $Z_{e}=2 \mathrm{~g}$, a clear softening type response becomes evident and the linear model becomes highly inaccurate. Steady-state transverse oscillations measured at the location of the laser vibrometer reading $\left(w_{s s}^{*}\right)$ are 

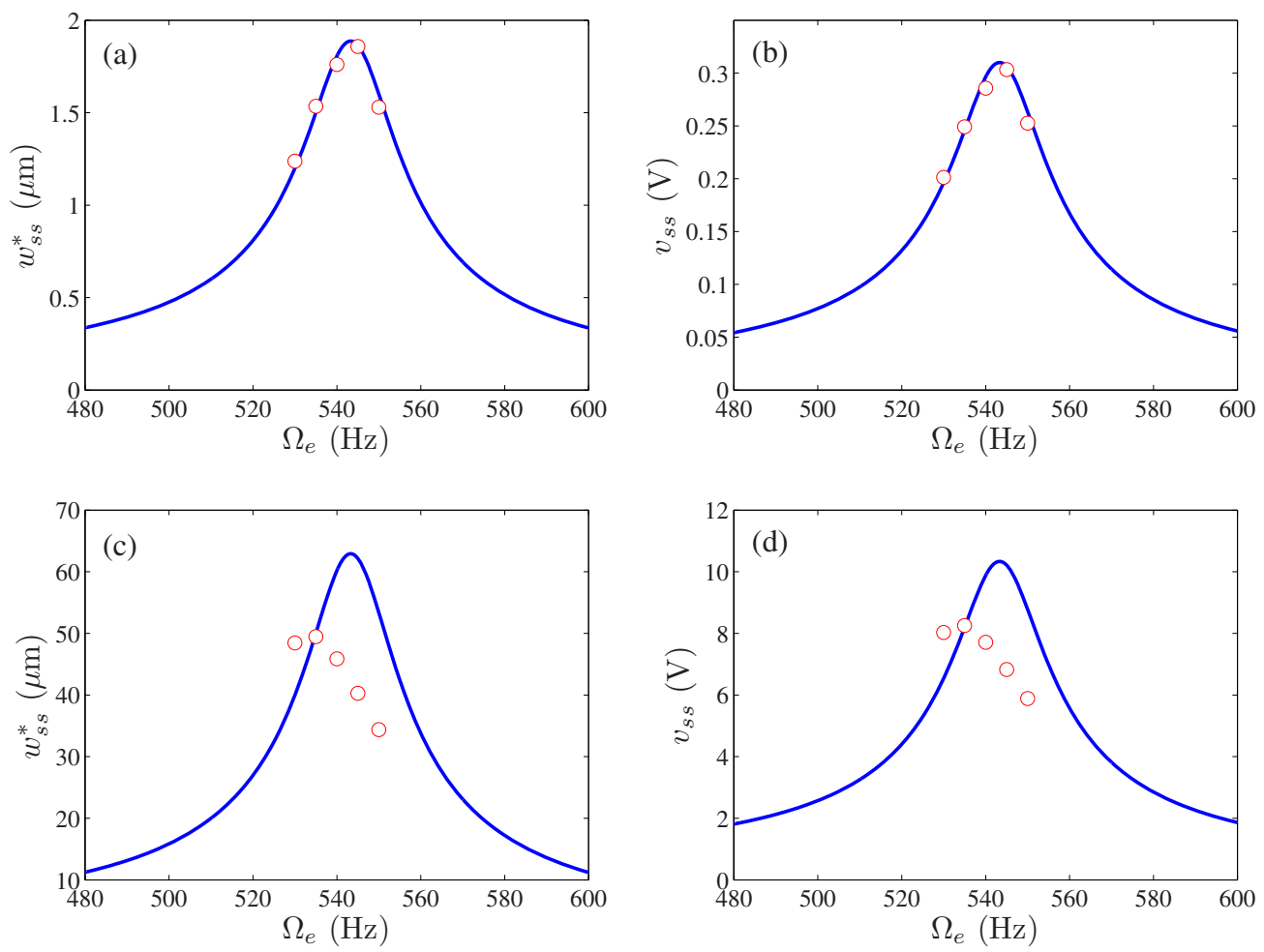

FIG. 3. (Color online) Good theoretical agreement with the linear piezoelectric model for low base acceleration $Z_{e}=60 \mathrm{mg}$ in the deflection (a) and voltage (b). For $Z_{e}=2 \mathrm{~g}$, however, (c) and (d) illustrate the inadequacy of the linear framework.

on the order of micrometers and are well within the elastically linear regime. Therefore, piezoelectric nonlinearities are adequately engaged.

The steady state voltage $v_{s s}$ and displacement $w_{s s}^{*}$ ampli-

(a)

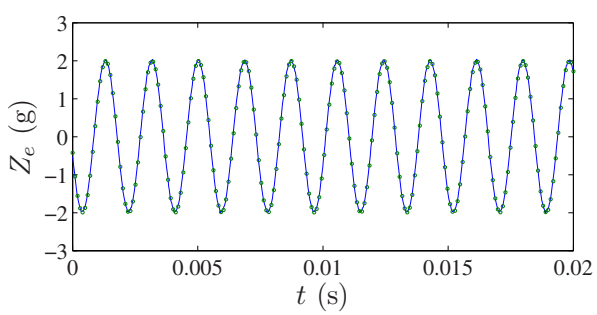

(c)

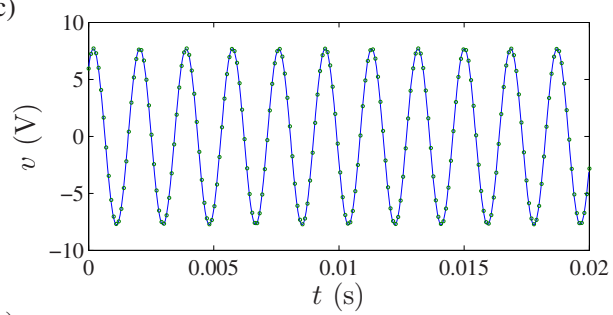

(e)

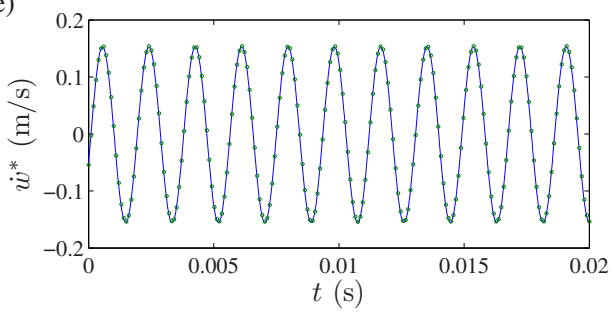

tudes were determined by Fourier transform techniques upon $60 \mathrm{~s}$ of transient-free data. For $Z_{e}=2 \mathrm{~g}$, the left column of Fig. 4 shows the experimental time series and reconstructed signals using the amplitude of the first harmonic only for the

(b)

(d)
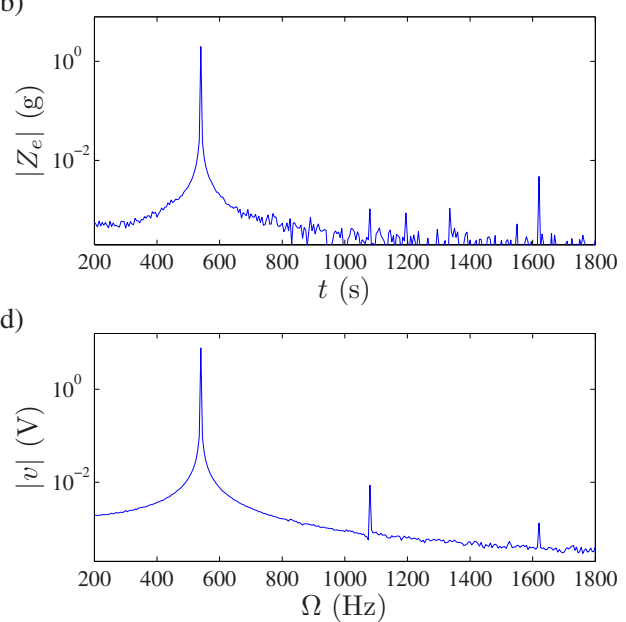

(f)

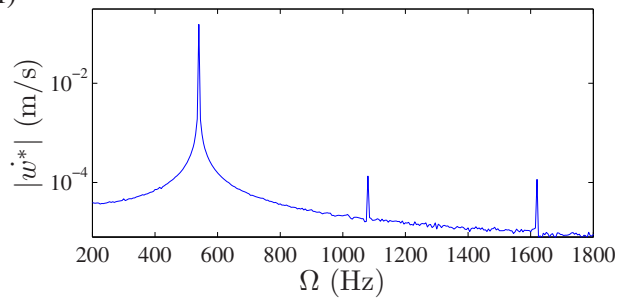

FIG. 4. (Color online) Experimental time series data (left column) and corresponding frequency content (right column). In (a), (c), and (f), experimental measurements are given by the solid line while the circles are points for a reconstructed signal using the amplitude of the first harmonic only. All data is for $Z_{e}=2 \mathrm{~g}$ and $\Omega_{e}=540 \mathrm{~Hz}$. 
base acceleration, beam tip velocity, and voltage. The corresponding amplitude of the transformed data in the frequency domain is shown in the right column of Fig. 4. Even though most of the signal energy is within the first harmonic as illustrated by the agreement shown in the first column, there exists expected signal energy at odd superharmonics of the excitation frequency but additional unanticipated signal energy at even superhamonics as well. Asymmetric configurations yield quadratic nonlinearities and even small imperfections in a seemingly symmetric structure can amplify the role of quadratic terms an thus produce signal energy at twice the forcing frequency. Considering the excellent agreement between the linear FRFs and the experimental data at weak excitation, asymmetry in the experimental beam was assumed insignificant. As an initial attempt, this study will utilize the equations of motion with cubic nonlinearity to provide the basis for which piezoelectric nonlinearity will be investigated. Future efforts will relax the presumption of an ideal symmetric configuration and thus introduce full consideration of both quadratic and cubic nonlinear influences.

Upon initial parameter identification trials, it was additionally observed that retaining only cubic nonlinearities would result in softening responses that would consistently overshoot the peak response in both the mechanical and electrical domains. Therefore, nonlinear damping was included in the theoretical model to account for the theoretical overshoot of experimental data. This explanation is further supported by the results of Malatkar and Nayfeh, ${ }^{50}$ who observed similar trends for the nonlinear response of a beam (see, in particular, Fig. 6 of Ref. 50).

\section{A. Parameter identification}

Equations (14) and (15) are reduced to a single mode approximation for parameter identification to facilitate an approximate analytic solution for implementation in an optimization routine. For harmonic excitation near a particular fundamental frequency, this simplification is sufficiently accurate for describing steady state oscillations. Also, since the potential difference across the resistive load is measured directly, it is convenient to re-express the flux linkage coordinates $\dot{\lambda}(t)$ and $\ddot{\lambda}(t)$ as $v(t)$ and $\dot{v}(t)$, respectively. Accordingly, with the addition of nonlinear damping, a reduced order model for the harvester is as follows:

$\ddot{x}+2 \zeta \omega \dot{x}+\zeta_{a} \dot{x}|\dot{x}|+\omega^{2} x+\alpha x^{3}-\left(\theta+\varphi x^{2}\right) v=\Gamma Z_{e} \cos \left(\Omega_{e} t\right)$,

$$
C \dot{v}+R^{-1} v+\left(\theta+\varphi x^{2}\right) \dot{x}=0,
$$

where $\omega$ is the first natural frequency of the cantilever and $\zeta_{a}$ is a nonlinear damping coefficient that is to be determined. Furthermore, the convenient result $\kappa_{111}=\varphi_{111} \equiv \varphi$ has been utilized. The model is next rendered dimensionless by defining a characteristic time, length, and voltage as $\tau=1 / \omega, \ell_{c}$ $=\Gamma Z_{e} \tau^{2}$, and $v_{c}=\ell_{c} \tau^{-1} C^{-1 / 2}$. With these parameters, Eqs. (17a) and (17b) take the form

$$
\ddot{x}+\mu_{1} \dot{x}+\mu_{2} \dot{x}|\dot{x}|+x+\beta x^{3}-\left(\sigma+\gamma x^{2}\right) \nu=\cos \Omega \tau,
$$

$$
\dot{\nu}+\mu_{3} \nu+\left(\sigma+\gamma x^{2}\right) \dot{x}=0 .
$$

This model is used from this point forward in our analysis. An approximate solution for the nonlinear amplitudefrequency relationship of the two degree-of-freedom model is found through the method of harmonic balance. Accordingly, we presume steady state solutions for the dimensionless modal displacement and voltage as

$$
\begin{aligned}
& x=a \cos \left(\Omega \tau+\phi_{x}\right), \\
& \nu=b \cos \left(\Omega \tau+\phi_{\nu}\right),
\end{aligned}
$$

where $\phi_{x}$ and $\phi_{v}$ is the respective phase offset. Following the technique originally proposed by Jacobsen, ${ }^{51}$ the nonlinear damping term is modeled as an equivalent viscous damping (see also Ravindra and Mallik ${ }^{52}$ )

$$
\mu_{2} \dot{x}|\dot{x}| \approx \mu_{2} \eta \Omega^{2} a^{2} \sin \left(\Omega \tau+\phi_{x}\right),
$$

where $\eta$ is a damping force ratio defined by

$$
\eta=\frac{4}{\pi} \int_{0}^{\pi / 2} \cos ^{3} u d u=\frac{2}{\sqrt{\pi}} \frac{\Gamma(2)}{\Gamma\left(\frac{5}{2}\right)},
$$

and $\Gamma$ above is the standard Gamma function. Inserting the result above along with the assumed solutions into Eqs. (18a) and (18b) and neglecting all but the first harmonic yields the set of equations

$$
\left(1-\Omega^{2}+\frac{3}{4} \beta a^{2}\right) a-b\left(\sigma+\frac{3}{4} \gamma a^{2}\right) \cos \left(\phi_{\nu}-\phi_{x}\right)=\cos \phi_{x},
$$

$$
\left(\mu_{1}+\frac{8}{3 \pi} \mu_{2} \Omega a\right) \Omega a-b\left(\sigma+\frac{1}{4} \gamma a^{2}\right) \sin \left(\phi_{\nu}-\phi_{x}\right)=\sin \phi_{x},
$$

$\left(\sigma+\frac{1}{4} \gamma a^{2}\right) \Omega a \sin \left(\phi_{\nu}-\phi_{x}\right)=\mu_{3} b$,

$\left(\sigma+\frac{1}{4} \gamma a^{2}\right) \Omega a \cos \left(\phi_{\nu}-\phi_{x}\right)=\Omega b$.

The voltage amplitude can be solved for by squaring and adding Eqs. (22c) and (22d) and the result used to solve for the phase difference relationships such that

$$
\begin{aligned}
& b=\frac{\Omega}{\sqrt{\mu_{3}^{2}+\Omega^{2}}}\left(\sigma+\frac{1}{4} \gamma a^{2}\right) a, \\
& \sin \left(\phi_{\nu}-\phi_{x}\right)=-\frac{\mu_{3}}{\sqrt{\mu_{3}^{2}+\Omega^{2}}}, \\
& \cos \left(\phi_{\nu}-\phi_{x}\right)=-\frac{\Omega}{\sqrt{\mu_{3}^{2}+\Omega^{2}}} .
\end{aligned}
$$

Substituting Eqs. (23a) and (23c) into Eqs. (22a) and (22b) and subsequently squaring and adding the result yields a tenth order polynomial expression for the steady state mechanical amplitudes as function of the excitation frequency 

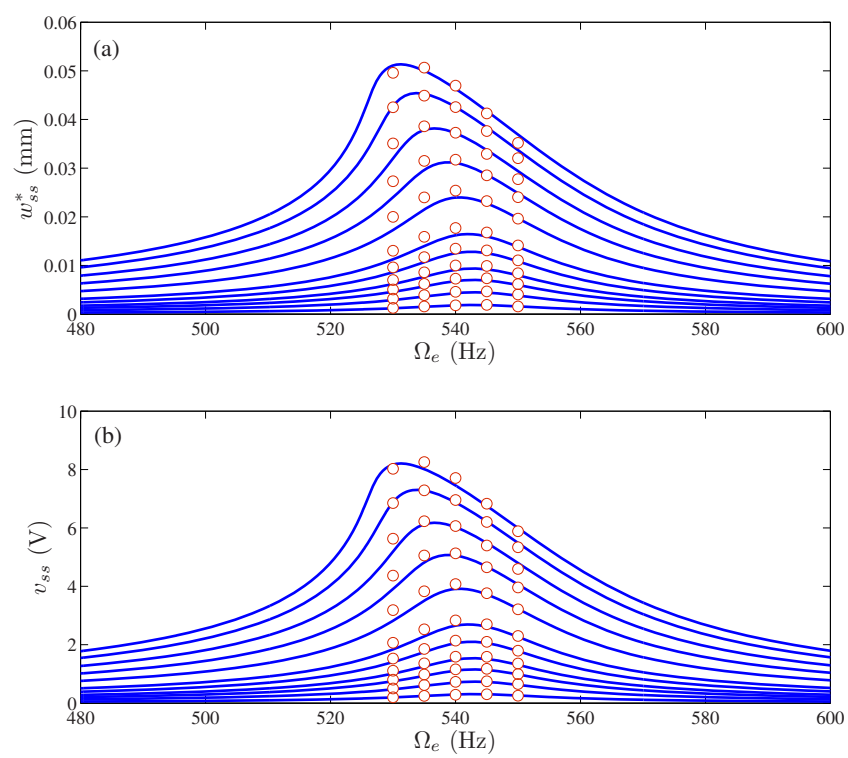

FIG. 5. (Color online) Theoretical agreement in both the mechanical response (a) and the voltage across the resistive load (b) upon optimizing nonlinear parameters to reflect experimental observations $(\mathrm{O})$.

$m_{10} a^{10}+m_{8} a^{8}+m_{7} a^{7}+m_{6} a^{6}+m_{5} a^{5}+m_{4} a^{4}+m_{3} a^{3}+m_{2} a^{2}$

$=1$,

where the $m_{j}$ terms are listed in the Appendix. Numerically resolving the roots of the nonlinear algebraic expression above for a range of excitation frequencies yields the amplitude dependent frequency response of the harvester.

A nonlinear least-squares algorithm, in particular LSQNONLIN in MATLAB, was used to find an optimal fit to the experimental data for the full range of excitation frequencies and amplitudes described in Sec. III. The following values were obtained for the unknown elastic, coupling, and damping nonlinearity coefficients expressed in dimensional terms as

$$
\begin{aligned}
& \alpha=-9.3722 \times 10^{17} \mathrm{~kg} / \mathrm{m}^{2} \mathrm{~s}^{2}, \\
& \varphi=-1.3642 \times 10^{10} \mathrm{C} / \mathrm{m}^{3}, \\
& \zeta_{a}=1.4038 \times 10^{4} \mathrm{~m}^{-1},
\end{aligned}
$$

which, upon considering Eqs. (16a) and (16c), yield the following higher order electroelasticity tensor values for PZT5H:

$$
\begin{aligned}
& c_{1111}^{p}=-3.6673 \times 10^{17} \frac{\mathrm{N}}{\mathrm{m}^{2}}, \\
& \text { and } e_{3111}=1.7212 \times 10^{8} \frac{\mathrm{m}}{\mathrm{V}} .
\end{aligned}
$$

The results for both the mechanical and voltage response are illustrated in Fig. 5, where the agreement can be seen to be quite good. Qualitatively, it was observed that the optimizing with an elastic nonlinearity alone would strongly shift the peak response to the left, undershooting the data recorded at $550 \mathrm{~Hz}$. Adding the coupling nonlinearity would begin to shift the nonlinear response back toward the right,
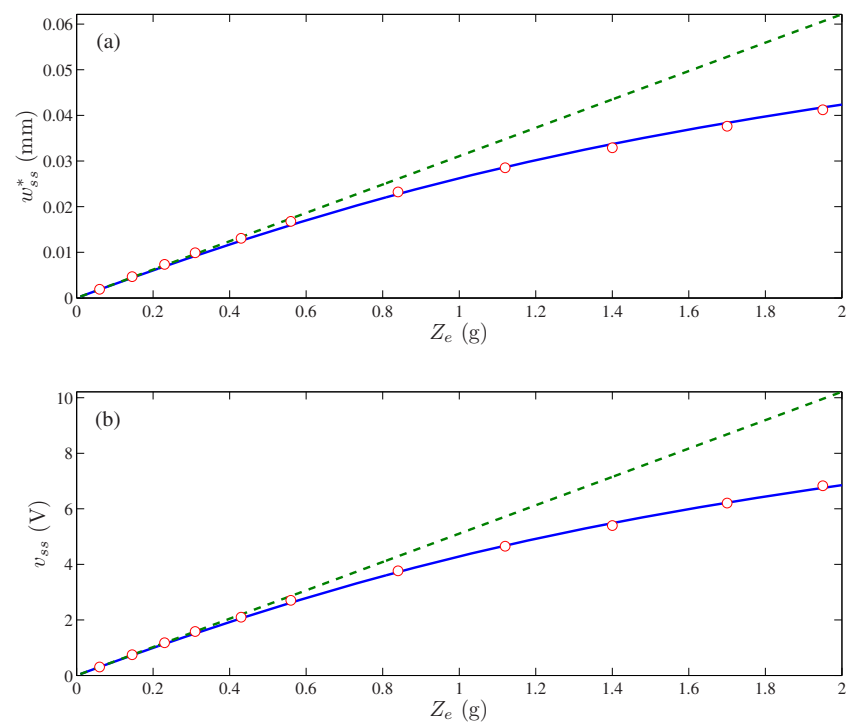

FIG. 6. (Color online) Comparison between linear theory (dotted line), nonlinear theory (solid line), and experimental measurements $(\mathrm{O})$ at a fixed excitation frequency $\Omega_{e}=545 \mathrm{~Hz}$ for the (a) mechanical response and (b) voltage across the resistive load.

but the peak response would continue to well overshoot the peak experimental values near $535 \mathrm{~Hz}$. The addition of quadratic nonlinear damping, however, was able to generate an optimal set of parameters for all nonlinear coefficients with very good agreement in both the mechanical and electrical response.

For an asymmetric microcantilever actuator, Mahmoodi et al. ${ }^{53}$ observed and modeled softening frequency response curves with a second order expansion of the linear constitutive laws. Cubic terms in their equations were a consequence of geometric nonlinearity that were significantly dominated by quadratic material nonlinearity terms due to the small size of the device. Since our device does not exhibit geometric nonlinear behavior and quadratic terms canceled due to symmetry, our results suggest that third order material nonlinearity was capable of inducing softening effects as well. However, we note that this modeling framework presumes an ideal symmetric configuration.

The shortcomings of the linear model in comparison to the new nonlinear model is shown by Fig. 6. Deviation from the linear model prediction occurs as early as $Z_{e}=0.5 \mathrm{~g}$ and further divergence underscores the importance of modeling inherent piezoelectric nonlinearity as well as nonlinear damping. Furthermore, since many piezoelectric harvesters are designed to operate at resonance, a linear model would suggest design parameters that do not reflect the frequency at which true peak response occurs. This is due to the softening effects as observed in this investigation. For example, Fig. 7 illustrates how the peak voltage response drifts in a nonlinear manner as base excitation is increased. This result cannot be predicted by the linear theory.

\section{SUMMARY AND CONCLUSIONS}

This paper investigated the manifestation and influence of nonlinearity on the dynamic response of geometrically linear piezoelectric energy harvester. A first-principles based 


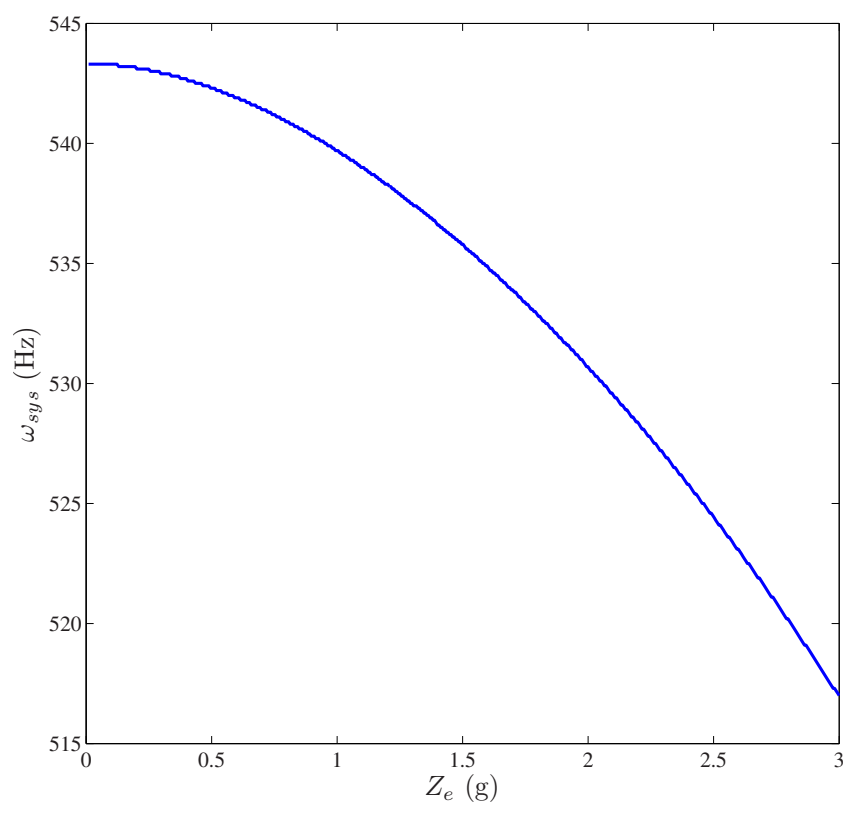

FIG. 7. (Color online) Frequency drift of the peak voltage response for varying base excitation.

model was derived using Hamilton's principle to physically justify nonlinear terms in the ensuing partial differential equations. Upon application of Galerkin's method and order reduction, a convenient set of ordinary differential equations for the harvester was established to facilitate derivation of an approximate analytic solution. The choice to add a quadratic dissipation term was rationalized based on frequency analysis of the experimental data. Third order nonlinearities in the equations of motion were a consequence of the cubic theory of nonlinear electroelasticity and the model suggests both that both elastic and electroelastic nonlinearities have a nontrivial influence on the response of the harvester. Parameter identification was accomplished by fitting a nonlinear algebraic equation derived via the method of harmonic balance with a nonlinear least-squares optimization algorithm. Good theoretical agreement with experimental data was demonstrated and fourth order tensor components for PZT-5H were suggested.

Most past work in energy harvesting has used linear theory to model experimentally observed behavior. Although an equivalent linear model can sometimes suffice to model the experimentally observed behavior over a very narrow range of excitations, substantial errors will arise when the excitation level is outside the narrow window unless the physical coefficients that describe the system are changed as well; however, there should be no reason why the physical parameters of the system change with excitation level. Proper inclusion of nonlinear terms in the physical models can be used to adequately capture the experimentally observed behavior.

Although the analysis herein focused on transverse oscillations of a cantilever with piezoceramics using $d_{31}$ poling, the authors strongly suspect similar results in $d_{33}$ poled piezostack type energy harvesters. This hypothesis is based on observations in Refs. 54 and 41 and is worthy of future investigation. The results presented in this paper are not lim- ited to energy harvesting applications and are applicable to any device utilizing piezoelectric cantilevers. Since nonlinear effects became discernible at fairly low base acceleration values, the results of this investigation underscore the necessity for inclusion of material nonlinearity in the performance assessment of piezoelectric energy harvesters operating near resonance. Future studies will more deeply explore the source of even superharmonics in the experimental system, which may involve re-examination of the presumption that electrostrictive behavior is negligible.

\section{ACKNOWLEDGMENTS}

Financial support from Dr. Ronald Joslin through an ONR Young Investigator Award is gratefully acknowledged as is support from AFOSR MURI Grant No. F-9550-06-10326, "Energy Harvesting and Storage Systems for Future Air Force Vehicles," monitored by Dr. B. L. Lee.

\section{APPENDIX: NONLINEAR COEFFICIENTS}

The coefficients of Eq. (24) are as follows:

$$
\begin{aligned}
m_{2}= & \frac{1}{\mu_{3}^{2}+\Omega^{2}}\left\{\mu_{3}^{2}\left[\left(\mu_{1}^{2}-2\right) \Omega^{2}+\Omega^{4}+1\right]\right. \\
& +\Omega^{2}\left[\Omega^{2}\left(\mu_{1}^{2}-2 \sigma^{2}-2\right)+\Omega^{4}+\left(\sigma^{2}+1\right)^{2}\right] \\
& \left.+2 \mu_{1} \mu_{3} \Omega^{2} \sigma^{2}\right\}, \\
m_{3}= & \frac{16 \mu_{2} \Omega^{3}\left[\mu_{1}\left(\mu_{3}^{2}+\Omega^{2}\right)+\mu_{3} \sigma^{2}\right]}{3 \pi\left(\mu_{3}^{2}+\Omega^{2}\right)} \\
m_{4}= & \frac{1}{18 \pi^{2}\left(\mu_{3}^{2}+\Omega^{2}\right)^{2}}\left[128 \mu_{2}^{2} \Omega^{4}\left(\mu_{3}^{2}+\Omega^{2}\right)^{2}\right. \\
& -9 \pi^{2}\left\{3 \beta\left(\mu_{3}^{2}+\Omega^{2}\right)\left[\mu_{3}^{2}\left(\Omega^{2}-1\right)+\Omega^{2}\left(\Omega^{2}-\sigma^{2}-1\right)\right]\right. \\
& -2 \Omega^{2} \sigma \gamma\left[\mu_{1} \mu_{3}^{3}+\mu_{1} \mu_{3} \Omega^{2}+\mu_{3}^{2}\left(-2 \Omega^{2}+\sigma^{2}+2\right)\right. \\
& \left.\left.\left.+2 \Omega^{2}\left(-\Omega^{2}+\sigma^{2}+1\right)\right]\right\}\right], \\
m_{10}= & \frac{\Omega^{2} \gamma^{4}\left(\mu_{3}^{2}+9 \Omega^{2}\right)}{256\left(\mu_{3}^{2}+\Omega^{2}\right)^{2}} . \\
m_{5}= & \frac{8 \mu_{2} \mu_{3} \Omega^{3} \sigma \gamma}{3 \pi\left(\mu_{3}^{2}+\Omega^{2}\right)}, \\
m_{6}= & \frac{\mu_{2} \mu_{3} \Omega^{3} \gamma^{2}}{16\left(\mu_{3}^{2}+\Omega^{2}\right)^{2}}\left\{9 \beta^{2}\left(\mu_{3}^{2}+\Omega^{2}\right)^{2}+24 \beta \Omega^{2} \sigma \gamma\left(\mu_{3}^{2}+\Omega^{2}\right)\right. \\
& +2 \Omega^{2} \gamma^{2}\left[\mu_{1} \mu_{3}^{3}+\mu_{1} \mu_{3} \Omega^{2}+\mu_{3}^{2}\left(-3 \Omega^{2}+3 \sigma^{2}+3\right)\right. \\
& \left.\left.+\Omega^{2}\left(-3 \Omega^{2}+11 \sigma^{2}+3\right)\right]\right\},
\end{aligned}
$$

${ }^{1}$ S. Beeby, M. Tudor, and N. White, Meas. Sci. Technol. 17, R175 (2006). 
${ }^{2}$ S. Anton and H. Sodano, Smart Mater. Struct. 16, R1 (2007).

${ }^{3}$ J. Kim, H. Takao, K. Sawada, and M. Ishida, Sensors 7, 1387 (2007).

${ }^{4}$ C. Enz, A. El-Hoiydi, J. Decotignie, and V. Peiris, Computer 37, 62 (2004).

${ }^{5}$ S. Roundy, J. Rabaey, and P. Wright, Comput. Commun. 26, 1131 (2003).

${ }^{6}$ G. Park, T. Rosing, M. Todd, C. Farrar, and W. Hodgkiss, J. Infrastruct. Syst. 14, 64 (2008).

${ }^{7}$ G. Ottman, H. Hofmann, A. Bhatt, and G. Lesieutre, IEEE Trans. Power Electron. 17, 669 (2002)

${ }^{8}$ R. Byrne and D. Diamond, Nature Mater. 5, 421 (2006).

${ }^{9}$ N. Dutoit, B. Wardle, and S.-G Kim, Integr. Ferroelectr. 71, 121 (2005).

${ }^{10}$ H. Sodano, G. Park, and D. Inman, Strain J. Brit. Soc. Strain Measurement 40, 49 (2004).

${ }^{11}$ H. Sodano and D. Inman, J. Intell. Mater. Syst. Struct. 16, 799 (2005).

${ }^{12}$ J. Scruggs, J. Sound Vib. 320, 707 (2009).

${ }^{13}$ J. Scruggs, "On the causal power generation limit for a vibratory energy harvester in broadband stochastic response," J. Intell. Mater. Syst. Struct. (in press).

${ }^{14}$ S. Stanton, Energy Harvesting from Turbulent Boundary Layer Pressure Fluctuations with Aeroacoustic Coupling: A Stochastic Optimal Control Approach, Master's thesis, Duke University, 2008.

${ }^{15}$ B. Mann and N. Sims, J. Sound Vib. 319, 515 (2009).

${ }^{16}$ B. Mann and B. Owens, J. Sound Vib. 329, 1215 (2010).

${ }^{17}$ F. Cottone, H. Vocca, and L. Gammaitoni, Phys. Rev. Lett. 102, 080601 (2009).

${ }^{18}$ L. Gammaitoni, I. Neri, and H. Vocca, Appl. Phys. Lett. 94, 164102 (2009).

${ }^{19}$ S. Stanton, C. McGehee, and B. Mann, Appl. Phys. Lett. 95, 174103 (2009).

${ }^{20}$ S. Stanton, C. McGehee, and B. Mann, Physica D 239, 640 (2010).

${ }^{21}$ A. Erturk, J. Hoffmann, and D. Inman, Appl. Phys. Lett. 94, 254102 (2009).

${ }^{22}$ D. Guyomar, A. Badel, E. Lefeuvre, and C. Richard, IEEE Trans. Ultrason. Ferroelectr. Freq. Control 52, 584 (2005).

${ }^{23}$ N. Hagood, W. Chung, and A. von Flotow, J. Intell. Mater. Syst. Struct. 1, 327 (1990).

${ }^{24}$ Q.-M Wang and L. E. Cross, IEEE Trans. Ultrason. Ferroelectr. Freq. Control 46, 1343 (1999).

${ }^{25}$ A. Erturk and D. Inman, Smart Mater. Struct. 18, 025009 (2009).

${ }^{26}$ A. Erturk and D. Inman, J. Vibr. Acoust. 130, 041002 (2008).
${ }^{27}$ A. Erturk and D. Inman, J. Intell. Mater. Syst. Struct. 19, 1311 (2008).

${ }^{28}$ A. Erturk and D. Inman, Smart Mater. Struct. 17, 065016 (2008).

${ }^{29}$ A. Erturk and D. Inman, Smart Mater. Struct. 17, 058001 (2008).

${ }^{30}$ Y. Hu, H. Xue, and J. Yang, IEEE Trans. Ultrason. Ferroelectr. Freq. Control 53, 1387 (2006).

${ }^{31} \mathrm{G}$. Maugin, Nonlinear Electromechanical Effects and Applications (World Scientific, Singapore, 1985).

${ }^{32}$ J. Yang, Analysis of Piezoelectric Devices (Springer, New York, 2005).

${ }^{33}$ H. Tiersten, J. Appl. Phys. 74, 3389 (1993).

${ }^{34}$ K. Wolf and O. Gottlieb, J. Appl. Phys. 91, 4701 (2002).

${ }^{35} \mathrm{~K}$. Wolf and O. Gottlieb, Technicon-Israel Institute of Technology, Technical Report No. ETR-2001-02, 2001 (http://meeng.technion.ac.il/ Research/TReports/2001/ETR-2001-02.pdf).

${ }^{36}$ T. Usher and A. Sim, J. Appl. Phys. 98, 064102 (2005).

${ }^{37}$ A. Triplett and D. Quinn, J. Intell. Mater. Syst. Struct. 20, 1959 (2009).

${ }^{38}$ E. Crawley and E. Anderson, J. Intell. Mater. Syst. Struct. 1, 4 (1990).

${ }^{39}$ E. Crawley and K. Lazarus, AIAA J. 29, 944 (1991).

${ }^{40}$ U. von Wagner and P. Hagendorn, J. Sound Vib. 256, 861 (2002).

${ }^{41}$ U. von Wagner and P. Hagendorn, Nonlinear Dyn. 31, 133 (2003).

${ }^{42}$ M. Samal, P. Seshu, S. Parashar, U. von Wagner, P. Hagendorn, B. Dutta, and H. Kushwaha, Finite Elem. Anal. Design 41, 1464 (2005).

${ }^{43}$ S. Stanton and B. Mann, "Nonlinear electromechanical dynamics of piezoelectric inertial generators: Modeling, experiment, and analysis," Nonlinear Dyn. (to be published).

${ }^{44}$ D. Guyomar, N. Aurelle, and L. Eyraud, J. Phys. III 7, 1197 (1997).

${ }^{45}$ S. Crandall, D. Karnopp, E. Kurtz, and D. Pridmore-Brown, Dynamics of Mechanical and Electromechanical Systems (Krieger, Malabar, FL, 1968).

${ }^{46}$ A. Tabesh and L. Frechette, J. Micromech. Microeng. 18, 104009 (2008).

${ }^{47}$ T. Low and W. Guo, J. Microelectromech. Syst. 4, 230 (1995).

${ }^{48}$ L. Meirovitch, Fundamentals of Vibrations (Wiley, New York, 2001).

${ }^{49}$ R. Massana and M. Daqaq, "Electromechanical modeling and nonlinear analysis of axially-loaded energy harvesters," J. Vibr. Acoust. (to be published).

${ }^{50}$ P. Malatkar and A. Nayfeh, J. Vib. Control 9, 317 (2003).

${ }^{51}$ L. Jacobsen, Trans. ASME 52, 169 (1930).

${ }^{52}$ B. Ravindra and A. Mallik, Phys. Rev. E 49, 4950 (1994).

${ }^{53}$ S. Mahmoodi, N. Jalili, and M. Daqaq, IEEE/ASME Trans. Mechatron. 13, 58 (2008).

${ }^{54}$ U. von Wagner, Int. J. Non-linear Mech. 38, 565 (2003). 